Article

\title{
Design of Hybrid Phase Sliding Mode Control Scheme for Lower Extremity Exoskeleton
}

\author{
Lingling Chen ${ }^{1,2}$, Chao Wang ${ }^{1}\left(\mathbb{D}\right.$, Jie Wang ${ }^{1,2, *}$ and Xiaowei Song ${ }^{1}(\mathbb{C}$ \\ 1 School of Artificial Intelligence, Hebei University of Technology, Tianjin 300130, China; \\ chenling@hebut.edu.cn (L.C.); 201732502020@stu.hebut.edu.cn (C.W.); 15122166812@163.com (X.S.) \\ 2 Engineering Research Center of Intelligent Rehabilitation, Ministry of Education, Tianjin 300130, China \\ * Correspondence: wangjie@hebut.edu.cn
}

Received: 28 June 2019; Accepted: 4 September 2019; Published: 8 September 2019

\begin{abstract}
Aiming at a pneumatic artificial muscle (PAM) lower extremity exoskeleton, a control mechanism based on hybrid phase sliding mode control (SMC) is proposed. First of all, the human gait cycle is mainly divided into the swing phase and stance phase, and the lower extremity exoskeleton phase models are established by the Euler-Lagrange method, respectively. Secondly, the lower limb exoskeleton is inevitably affected in the diverse working environment, and the exoskeleton model has nonlinear and strong coupling characteristics, which both increase the control difficulty. In this situations, a robust sliding mode control method is designed based on an Extended State Observer (ESO). Thirdly, the pneumatic muscle takes time to contract and relax, and then the joint input torque cannot jump when the gait phase changes, hence, the smoothing switching of the assistive control scheme is introduced to solve it. The smoothing switching time is detected by a phase detector, and the phase detector is designed by the plantar pressure information. Finally the comparative simulation shows that this control strategy has the advantages of fast time, high control precision and no jump during control torque switching. Pneumatic artificial muscle contraction rate curve shows that the pneumatic muscles' motion range meets the control requirement of the exoskeleton.
\end{abstract}

Keywords: lower extremity exoskeleton; pneumatic artificial muscle; gait switching; extended state observer; sliding mode control

\section{Introduction}

The lower extremity exoskeleton is a wearable device with an active actuator that provides active torque to the operator and helps the operator to accomplish subjective willing movements. Exoskeletons can be used in many fields, such as military [1,2], construction $[3,4]$, service $[5,6]$, and medical [7-9], which have attracted scholars' extensive attention from all over the world [10]. In recent decades, different research institutions have adopted different control mechanisms for the exoskeleton [11,12], the Hybrid Control Strategy [13] among them is applied in exoskeleton systems for Human-Performance and obtains improved performance successfully. In BLEEX, a hybrid control strategy [14] is put forward to control the lower limb exoskeleton in the swing phase or stance phase respectively, where the sensitivity amplification controller (SAC) is employed for the swing leg, the position control strategy is applied for the stance leg. Another Hybrid Control Strategy is presented to control a hydraulically actuated lower limb exoskeleton, where the $H_{\infty}$ control method is designed to improve the performance under all kinds of uncertainties. Besides, the control algorithm for switching is used to reduce the delay of exoskeleton tracking [15]. For a Load-Carrying lower extremity exoskeleton, the position control approach is applied in the stance phase, and the following control method is used in the swing phase [16]. In [17], for different dynamic models of a gait 
cycle, a hybrid phase control method is described to determine the phase by the Back F/T sensor and Foot F/T sensor. In [18], a control mechanism is proposed, where the stance phase adopts active control, the swing phase then switchs to follow-up control, and the foot sensor performs the phase discrimination.

Most hybrid control strategies adopts a phase-dependent control strategy to control the corresponding phase model, which usually depends on the characteristics of the lower extremity exoskeleton. The lower extremity exoskeleton model has the nonlinear and strong coupling characteristics [19], in addition, the exoskeleton systems exist uncertain disturbances such as parameter changes and extern interference caused by the working environment. Hence, it is not suitable to achieve excellent control performance by applying traditional control methods [20]. In [21], the adaptive iterative learning control (AILC) method is proposed, which can effectively reduce such error without sacrificing the iteration efficiency. In [22], a model-free control method is presented to estimate the knee joint angles of the exoskeleton. Then, to improve the control precision and robustness of the system, the discrete sliding mode control law and prior torque are introduced. In [23] , an improved single-input fuzzy sliding mode controller together with an adaptive switching controller is proposed to promote the tracking performance of the system. Because the system is affected by interference, the rejection control [24] is regarded to overcome the active interference and applied it to track the human gait trajectory, in that the extended state observer is designed to estimate the total uncertain disturbances. In [25], the sliding mode controller based on the extended state observer is proposed to solve the tracking control problem of the double joint dexterous hand and improve control performance when compares with a single sliding mode controller.

With aforementioned, this article focus on the hybrid phase control and the phase switching problem. The block diagram of the whole control system is shown in Figure 1. The main innovations are as follows: first, assuming that the human body moves at a constant speed in the horizontal direction, according to that pneumatic lower extremity exoskeleton structure, the dynamic models are established in the swing phase and stance phase respectively using the Euler-Lagrange formulation. After that, the SMC based on ESO is designed to fit to the working environment and characteristics of lower extremity exoskeleton. The tracking speed of the system can be increased and the total disturbances can be estimated. Then, the plantar pressure data collection system is built by High Dynamic Force Sensing Resistor-Insole together with the stm 32 microcontroller, and the phase detector is designed based on the data to provide a switching time. Finally, a smoothing mechanism is adopted to solve the sudden change (command jump) of the control torque, which meets the job condition of the artificial pneumatic muscle during the switching process.

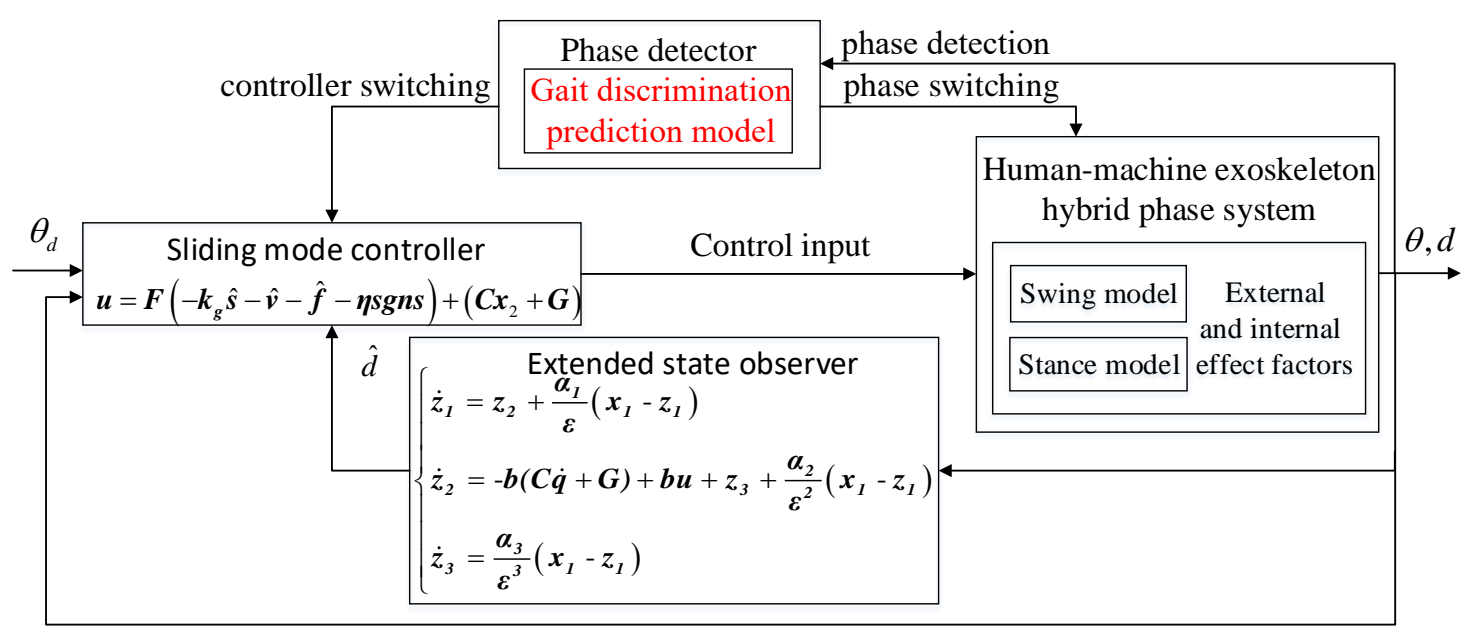

Figure 1. Control strategy block diagram for the exoskeleton in hybrid phase. 
The rest of the paper is organized as follows. Section 2 presents a parameterized model of lower extremity exoskeleton, which is established by the Lagrange dynamic method. An unconventional sliding mode controller based on the high gain extend state observer is proposed, the stability of the system is proved by the Lyapunov function. Based on plantar pressure data, the gait division and phase detector are accomplished, then the smoothing function is adopted. Section 3 demonstrates the results of the comparative simulations and partial experiments. Section 4 summarizes the conclusions and potential future work.

\section{Methods}

\subsection{Dynamic Model of Lower Extremity Exoskeleton}

As shown in Figure 2, a numerical characterization method [26,27] of the lower limbs is adopted to obtain the switching model in the sagittal plane. In this method, it is assumed the lower limbs of the human body usually perform the periodic motion, and the human legs are symmetrical. Therefore, the simplified model can be seen as a double link, which is towed by pneumatic muscles. In the swing phase, the oscillating leg rotates around the hip joint $P_{0}\left(x_{0}, y_{0}\right)$; During the stance phase, the thigh and upper torso are approximated as the fixed axis motion rotating around the foot, the axis is between the foot and the ground $P_{0}\left(x_{0}, y_{0}\right)$, and $P_{16}$ represents the center of mass of the torso. The schematic diagram of the lower extremity exoskeleton is shown in Figure 2 , and $P_{11} P_{14}, P_{1} P_{15}$, $P_{10} P_{8}$ are corresponding to three pneumatic muscles.

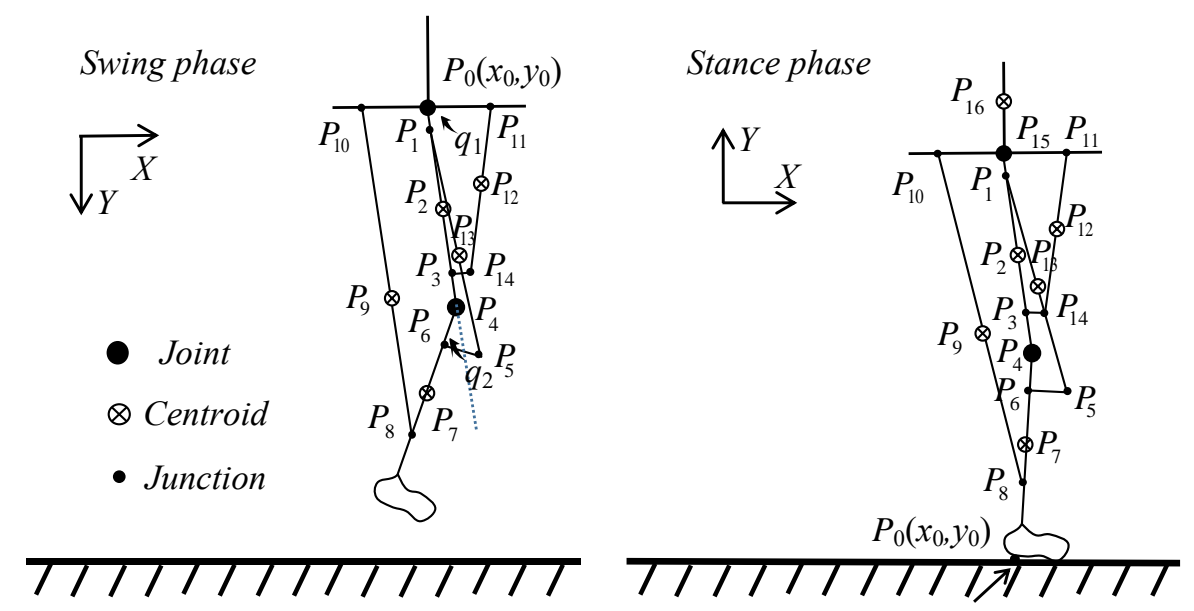

Figure 2. Swing phase model (Left) and Stance phase model (Right).

The meaning of the symbols in Figure 2 is shown as follows:

- $\quad P_{4}$ : The knee joint

- $\quad P_{0}$ : The hip joint/The foothold point

- $\quad P_{5}$ : The end of the pole fixed on the calf

- $\quad P_{8}$ : The connection point between pneumatic muscle 3 and calf

- $\quad P_{1}, P_{3}$ : The connection point between pneumatic muscle 1,2 and thigh

- $\quad P_{10}, P_{11}$ : The connection point between pneumatic muscle 1,3 and waist

- $m_{1}, m_{2}:$ The quality of thigh and calf

- $m_{6}$ : The quality of the human upper body

- $m_{3}, m_{4}, m_{5}$ : The quality of pneumatic muscle 1-3

- $\quad r_{1}$ : The length between $P_{1}$ and $P_{0}$

- $\quad r_{2}$ : The length between $P_{3}$ and $P_{0}$

- $\quad r_{3}$ : The length between $P_{4}$ and $P_{6}$ 
- $\quad r_{4}$ : The length between $P_{4}$ and $P_{8}$

- $\quad l_{1}, l_{2}$ : The length between $P_{10}, P_{11}$ and $P_{0}$

- $q_{1}, q_{2}$ : The angle of hip and knee

Based on the mention above, the model of the single-leg during the swing and stance phase are respectively accomplished by using the Lagrange dynamics method, the general hybrid phase dynamics of the lower extremity exoskeleton system can be written as follows (See Appendix A for details):

$$
F_{i}(q) \ddot{q}+C_{i}(q, \dot{q}) \dot{q}+G_{i}(q)=\tau_{a c t}+\tau_{h m}
$$

where $q$ is the angle vector of the joints, $F(q)$ is a inertia matrix and a function of $q, C(q, \dot{q})$ a centripetal and Coriolis matrix, $G(q)$ is a vector of gravitational torque. $\tau_{\text {act }}$ is the actuator torque vector and $\tau_{h m}$ may contain two part: $\tau_{1}=J^{T} F_{1}$ and $\tau_{2}=J^{T} F_{2} . F_{1}$ is the interactive force of the human body acting on the exoskeleton and $F_{2}$ is the contact force provided by the ground only in the stance phase. They can be transformed into control torque on the joints through the Jacobian matrix $J . \tau_{1}, \tau_{2}$ can be seen as the extern interference for exoskeleton system. The value of subscript can be selected as 1 or 2 , which represent the swing phase and the stance phase respectively. Equation (1) can be deduced to:

$$
\left\{\begin{array}{l}
\dot{x}_{1}=x_{2} \\
\dot{x}_{2}=-b(C \dot{q}+G)+b u-b d \\
y=x_{1}
\end{array}\right.
$$

where $x_{1}=q=\left[\begin{array}{ll}q_{1} & q_{2}\end{array}\right]^{\mathbf{T}}, x_{2}=\dot{q}=\left[\begin{array}{ll}\dot{q}_{1} & \dot{q}_{2}\end{array}\right]^{\mathbf{T}}$, the output of exoskeleton system is denoted as $y \in R^{2 \times 1} . q, \dot{q}, \ddot{q} \in R^{2 \times 1}$ denote the joint angle, angular velocity and angular acceleration of the lower extremity exoskeleton. $b=F^{-1}$ is a nonlinear smoothing function and the system disturbances are denoted by $d$. The input torque of the hip and knee are $u=\tau \in R^{2 \times 1}$. Hence, the system can be rewritten as multiple-input multiple-output (MIMO) system. The disturbance item includes system internal variable parameters and external interference:

$$
f=-b d=F^{-1}\left(\tau_{h m}-(\Delta F \ddot{q}+\Delta C \dot{q}+\Delta G)\right) \in R^{n}
$$

where $\Delta F, \Delta C$ and $\Delta G$ represent variable parameters and model errors. Equation (3) satisfies the following proposition:

Proposition 1. The derivatives of Equation (3) exist and satisfy $\|\dot{f}\| \leq D$, and $D>0$ is a known constant.

\subsection{Design of SMC Based on ESO}

\subsubsection{Design of Extended State Observer}

In this section, ESO is designed, which estimates the total disturbances. First of all, an additional lemma is introduced to prove the convergence of ESO.

Lemma 1. For any symmetric positive definite matrix, there is a positive definite matrix that satisfies $\bar{A}^{T} P+P \bar{A}=-Q$.

For the system (2), the following extended state observer is designed:

$$
\left\{\begin{array}{l}
\dot{z}_{1}=z_{2}+\frac{\alpha_{1}}{\varepsilon}\left(x_{1}-z_{1}\right) \\
\dot{z}_{2}=-b(C \dot{q}+G)+b u+z_{3}+\frac{\alpha_{2}}{\varepsilon^{2}}\left(x_{1}-z_{1}\right) \\
\dot{z}_{3}=\frac{\alpha_{3}}{\varepsilon^{3}}\left(x_{1}-z_{1}\right)
\end{array}\right.
$$


where $z_{1}, z_{2}, z_{3} \in R^{2 \times 1}$ are states of the observer system. $\alpha_{1}, \alpha_{2}, \alpha_{3}$ are the gain matrixs of the observer, and $\varepsilon \in R$ is observer normal constant parameter to be designed.

$$
\left\{\begin{array}{l}
\alpha_{1}=\operatorname{diag}\left(l_{11}, l_{12}\right) \\
\alpha_{2}=\operatorname{diag}\left(l_{21}, l_{22}\right) \\
\alpha_{3}=\operatorname{diag}\left(l_{31}, l_{32}\right)
\end{array}\right.
$$

where $l_{i j}>0,(i=1,2,3 ; j=1,2)$.

Theorem 1. Under the proposition 1 and the lemma 1, with the lower extremity exoskeleton robot system (2), the high gain extended state observer is given by (4), the total disturbances are observed by state $z_{3}$, and the upper limit of the observation error depends on the positive constant parameter $\varepsilon$.

Proof of Theorem 1. Define the observer error is:

$$
\xi=\left[\xi_{1}, \xi_{2}, \xi_{3}\right]^{T}
$$

where $\xi_{1}=\frac{x_{1}-z_{1}}{\varepsilon^{2}}, \xi_{2}=\frac{x_{2}-z_{2}}{\varepsilon}, \xi_{3}=f(t)-z_{3}$.

Deriving on both sides of Equation (6) and multiply it with $\varepsilon$,

$$
\left\{\begin{array}{c}
\varepsilon \dot{\xi}_{1}=-\alpha_{1} \xi_{1}+\xi_{2} \\
\varepsilon \dot{\xi}_{2}=-\alpha_{2} \tilde{\xi}_{1}+\tilde{\xi}_{3} \\
\varepsilon \dot{\xi}_{3}=-\alpha_{3} \xi_{1}+\varepsilon \dot{f}(t)
\end{array}\right.
$$

Consider the observer canonical form realization, $\bar{A}=\left[\begin{array}{ccc}-\alpha_{1} & I_{2 \times 2} & 0_{2 \times 2} \\ -\alpha_{2} & 0_{2 \times 2} & I_{2 \times 2} \\ -\alpha_{3} & 0_{2 \times 2} & 0_{2 \times 2}\end{array}\right], \bar{B}=\left[\begin{array}{c}0_{2 \times 2} \\ 0_{2 \times 2} \\ I_{2 \times 2}\end{array}\right]$, and the observer error equation obtained:

$$
\varepsilon \dot{\xi}=\bar{A} \xi+\varepsilon \bar{B} \dot{f}
$$

Define the observer Lyapunov function is:

$$
V_{o}=\varepsilon \xi^{T} P \xi
$$

Deriving on both sides of the above Equation (9)

$$
\begin{aligned}
\dot{V}_{o} & =\varepsilon \dot{\zeta}^{T} P \xi+\varepsilon \xi^{T} P \dot{\xi}=(\bar{A} \xi+\varepsilon \bar{B} \dot{f})^{T} P x+\xi^{T} P(\bar{A} \xi+\varepsilon \bar{B} \dot{f}) \\
& =\left(\xi^{T} \bar{A}^{T}+\varepsilon(\bar{B} \dot{f})^{T}\right) P \xi+\xi^{T} P(\bar{A} \xi+\varepsilon \bar{B} \dot{f}) \\
& =\xi^{T}\left(\bar{A}^{T} P+P \bar{A}\right) \xi+\varepsilon\left[(\bar{B} \dot{f})^{T} P \xi+\xi^{T} P(\bar{B} \dot{f})\right] \\
& =\xi^{T}\left(\bar{A}^{T} P+P \bar{A}\right) \xi+2 \varepsilon \xi^{T} P \bar{B} \dot{f} \leq-\xi^{T} Q \xi+2 \varepsilon D\|P \bar{B}\|\left\|\xi^{T}\right\|
\end{aligned}
$$

By Lemma 1, Equation (10) can be rewritten as

$$
\dot{V}_{o} \leq-\lambda_{\min }(Q)\|\xi\|^{2}+2 \varepsilon D\|P \bar{B}\|\|\xi\|
$$

where $\lambda_{\min }$ is the minimum eigenvalue. Eventually, the convergence condition of the observer is

$$
\|\xi\| \leq \frac{2 \varepsilon D\|P \bar{B}\|}{\lambda_{\min }(Q)}
$$


Equation (12) indicates: the system observation error has convergence upper bound, and the final error depends on the value of the parameter $\varepsilon$. The smaller the selected $\varepsilon$, the smaller the observation error.

\subsubsection{Design of Sliding Mode Controller}

This work considers the nonlinear and strong coupling characteristics of the exoskeleton and designs the robust sliding mode controller combining with the system (2). The proof step of the controller does not directly use the upper limit of the disturbance but relies on the upper limit of the observer's observation error. As long as the observer has good observation ability (observation error converges) and ensures that the observation has an upper bound, then the proof of the controller is valid. Considering the stability of the system under the extended state observer (4), the controller can be proposed. The following lemma and proposition should be pointed out in advance:

Lemma 2. For the candidate Lyapunov function $V:[0, \infty) \in R$, the solution of the inequality equation $\dot{V} \leq-\alpha V+f, \forall t \geq t_{0} \geq 0$ is

$$
V(t) \leq e^{-\alpha\left(t-t_{0}\right)} V\left(t_{0}\right)+\int_{t_{0}}^{t} e^{-\alpha(t-\tau)} f(\tau) \mathrm{d} \tau
$$

Proposition 2. The observation error under (4) is limited, that is $\left\|\tilde{v}+\tilde{f}+k_{g} \tilde{s}\right\| \leq \Delta_{\max }, \Delta_{\max }>0$.

The reference position is indicated by $x_{1 d}=\left[q_{1 d}, q_{2 d}\right]^{T}$, define the tracking error as

$$
e=x_{1}-x_{1 d}=\left[e_{1}, e_{2}\right]^{\mathrm{T}}
$$

The sliding mode surface is defined as

$$
s=c e+\dot{e}, c=\operatorname{diag}\left(c_{1}, c_{2}\right)
$$

According to Equation (15) and system (2), the general sliding mode controller has the following form:

$$
u=F\left(-c \dot{e}+\ddot{\theta}_{d}-\eta \operatorname{sgn} s\right)-D \operatorname{sgn} s+\left(C x_{2}+G\right)
$$

where $\eta>0$ denotes the switching gain, $D$ is considered to be the upper bound of disturbances, that is $|d| \leq D$, the conventional sliding mode control often requires large switching gains to eliminate extern disturbances and uncertainties. However, the large switching gains will cause chattering. Through the designed extended state observer, the disturbances estimation are used as the feed-forward input of the controller, which greatly reduces the gain of the switching term in the sliding mode controller and effectively eliminates chattering. The sliding mode controller is designed as follows:

$$
u=F\left(-k_{g} \hat{s}-\hat{v}-\hat{f}-\eta \operatorname{sgn} s\right)+\left(C x_{2}+G\right)
$$

where

$$
\begin{aligned}
& k_{g}=\operatorname{diag}\left(k_{1}, k_{2}\right), \quad k_{1}, k_{2}>0, \\
& \hat{v}=\hat{\dot{e}}-\ddot{x}_{1 d}, \hat{s}=c \hat{e}+\hat{e}, \hat{e}=\hat{x}_{1}-x_{1 d}, \\
& \hat{e}=\hat{x}_{2}-x_{2 d}, \\
& \tilde{v}=v-\hat{v}, \tilde{f}=f-\hat{f}, \tilde{s}=s-\hat{s} .
\end{aligned}
$$

Theorem 2. Consider the exoskeleton system (2) and the extended state observer(4), the system globally stable under the controller(17) if the proposition 1-2 are satisfied and Lemma 1-2 are applied. The tracking error has an upper bound of convergence, and the convergence speed depends on positive constant parameter $\varepsilon$ and control gain $k_{g}$. 
Proof of Theorem 2. Consider a candidate Lyapunov function $V_{c}=\frac{1}{2} s^{T} s$, and differentiating both sides of $V_{c}$ :

$$
\begin{aligned}
\dot{V}_{c} & =s^{T} \dot{s}=s^{T}\left(c \dot{e}+b u+f-\ddot{x}_{1 d}-b\left(C x_{2}+G\right)\right) \\
& =s^{T}\left(c \dot{e}-k_{g} \hat{s}-\hat{v}-\hat{f}+f-\ddot{x}_{1 d}-\eta \operatorname{sgn} s\right) \\
& =s^{T}\left(v-\hat{v}+f-\hat{f}-k_{g} \hat{s}-\eta \operatorname{sgn} s\right) \\
& =-s^{T} k_{g} \hat{s}+s^{T}(\tilde{v}+\tilde{f})-\eta|s| \\
& =-s^{T} k_{g}(s-\tilde{s})+s^{T}(\tilde{v}+\tilde{f})-\eta|s| \\
& =-s^{T} k_{g} s+s^{T}\left(\tilde{v}+\tilde{f}+k_{g} \tilde{s}\right)-\eta|s|
\end{aligned}
$$

where the observation errors are as follows:

$$
\begin{gathered}
\tilde{v}=v-\hat{v}=c \dot{e}-\ddot{x}_{1 d}-\hat{\dot{c} e}+\ddot{x}_{1 d}=c(\dot{e}-\hat{\dot{e}}) \\
=c\left(x_{2}-\dot{x}_{1 d}-\hat{x}_{2}+\dot{x}_{1 d}\right)=c\left(x_{2}-\hat{x}_{2}\right) \\
=c \tilde{x}_{2} \\
\tilde{s}=s-\hat{s}=c e+\dot{e}-c \hat{e}-\hat{e}=c(e-\hat{e})+(\dot{e}-\hat{\dot{e}}) \\
=c\left(x_{1}-x_{1 d}-\hat{x}_{1}+x_{1 d}\right)+\left(\dot{x}_{1}-\dot{x}_{1 d}-\hat{x}_{1}+\dot{x}_{1 d}\right) \\
=c \tilde{x}_{1}+\tilde{x}_{2}
\end{gathered}
$$

The Equation (18)-(21) indicate, $\tilde{v}+\tilde{f}+k_{g} \tilde{s}$ is depended on the state observation error $\tilde{x}_{1}=$ $\left[\tilde{q}_{1}, \tilde{q}_{2}\right], \tilde{x}_{2}=\left[\dot{q}_{1}, \dot{q}_{2}\right]$ of the system. Thus, $\Delta_{\max } \geq\left\|\tilde{v}+\tilde{f}+k_{g} \tilde{s}\right\|$ always satisfies,

$$
\begin{aligned}
& \dot{V}_{c} \leq-s^{T} k_{g} s+s^{T} \Delta_{\max } I_{2 \times 1}-\eta|s| \\
& \leq-s^{T} k_{g} s+\frac{1}{2}\left(s^{T} s+\Delta_{\max }^{2}\right)-\eta|s| \\
& \leq-\lambda_{\min }\left(2 k_{g}-I\right) \frac{1}{2} s^{T} s+\frac{1}{2} \Delta_{\max }^{2} \\
& =-\lambda_{\min }\left(2 k_{g}-I\right) V_{c}+\frac{1}{2} \Delta_{\max }^{2}
\end{aligned}
$$

Define $\alpha=-\lambda_{\min }\left(2 k_{g}-I\right), f=\frac{1}{2} \Delta_{\max }^{2}$, and then $V_{c}$ is calculated as follows based on Lemma 2:

$$
\begin{aligned}
V_{c} \leq & e^{-\alpha\left(t-t_{0}\right)} V_{c}\left(t_{0}\right)+\int_{t_{0}}^{t} e^{-\alpha(t-\tau)} f(\tau) d \tau \\
= & e^{-\lambda_{\min }\left(2 k_{g}-I\right)\left(t-t_{0}\right)} V_{c}\left(t_{0}\right)+\frac{1}{2} \Delta_{\max }^{2} \int_{t_{0}}^{t} e^{-\lambda_{\min }\left(2 k_{g}-I\right)(t-\tau)} d \tau \\
= & e^{-\lambda_{\min }\left(2 k_{g}-I\right)\left(t-t_{0}\right)} V_{c}\left(t_{0}\right)-\frac{1}{2 \lambda_{\min }\left(2 k_{g}-I\right)} \\
& \times \Delta_{\max }^{2} \int_{0}^{t-t_{0}} e^{-\lambda_{\min }\left(2 k_{g}-I\right)(t-\tau)} d\left(-\lambda_{\min }\left(2 k_{g}-I\right)(t-\tau)\right) \\
= & e^{-\lambda_{\min }\left(2 k_{g}-I\right)\left(t-t_{0}\right)} V_{c}\left(t_{0}\right)+\frac{1}{2 \lambda_{\min }\left(2 k_{g}-I\right)} \times \Delta_{\max }^{2}\left(1-e^{-\lambda_{\min }\left(2 k_{g}-I\right)\left(t-t_{0}\right)}\right)
\end{aligned}
$$

From analysis above, when $t \rightarrow \infty$, this is satisfying:

$$
V_{c}(t) \leq \frac{1}{2 \lambda_{\min }\left(2 k_{g}-I\right)} \Delta_{\max }^{2}
$$

where $k_{g}=\operatorname{diag}\left(k_{1}, k_{2}\right), k_{1}, k_{2}>\frac{1}{2}$. The convergence rate depends on controller gain $k_{g}$ and observer parameters $\varepsilon$. Finally, bring Equation (24) into Equation (22), $\dot{V}_{c} \leq 0$ can be confirmed. Thus, consider a closed loop system consisting of an observer and a controller, the Lyapunov function is $V=V_{o}+V_{c}$, the system is stable under the controller (17) when $k_{g}$ is big enough and $\varepsilon$ is small enough.

\subsection{Phase Analysis and Phase Switch}

\subsubsection{Phase Detector}

The hybrid phase control performs real-time detection of the human gait and then to determine the time of phase switching control. The phase detector created by Extreme Learning Machine can forecast gait before phase switching. The lower limb movement of the human body is generally divided into a 
swing phase and a stance phase. The single-leg gait is divided into several specific stages, as shown in Figure 3. The stance stage includes four states, and the swing stage includes three states which can be collectively referred to as the swing phase.

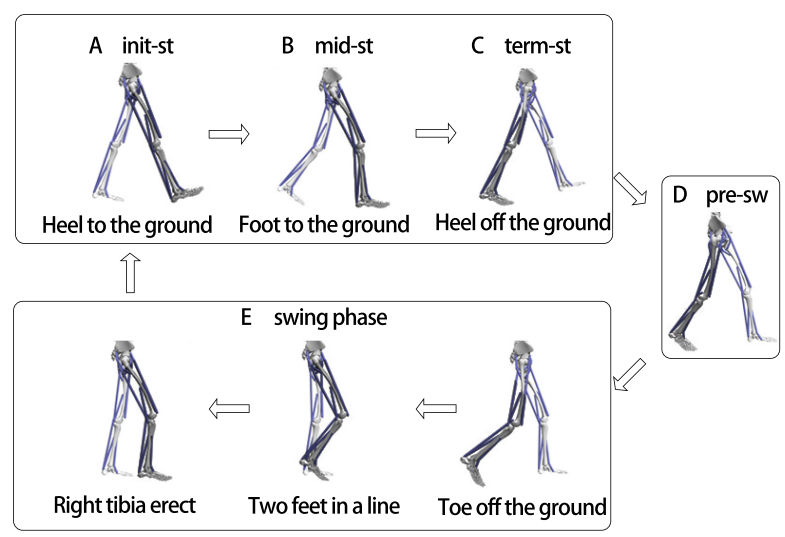

(a)

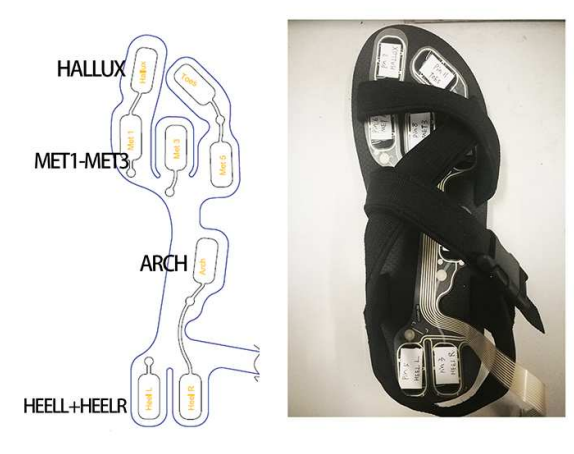

(b)

Figure 3. The left shows the division of seven gait phases, which represent the statistical results of the plantar pressure signal; the plantar pressure data is collected by the right High Dynamic Force Sensing Resistor-Insole. (a) Gait division; (b)High Dynamic Force Sensing Resistor-Insole.

The design of the plantar pressure measurement system is an important part of the hybrid phase control mechanism [28]. In this article, the plantar pressure is collecting by High Dynamic Force Sensing Resistor-Insole, which is pasted on the sandal. It has eight HD-FSR cells and can measure 7 channels punctual plantar pressure, including foot's heel, midfoot, metatarsal heads, and the toes. The AD module of the STM32F407 collects the plantar pressure data at a rate of $200 \mathrm{~Hz}$ and transmits the data to the host computer to display and store in real time via Bluetooth. In this experiment, 200 gaits cycles from 5 healthy subjects have been obtained. During data collection, it was found that the data of the TOES cell were not regular, and it was given up. The reason may be that the toes play a small role when the person walks. The data of HEELL and HEELR was averaged and considered to be the sampling of the heel. Therefore, High Dynamic Force Sensing Resistor-Insole was regarded as collecting six channels data at once time. The gait recognition method based on plantar pressure is the important content of switching. First, the gait characteristics are selected as the force characteristic and the force trend characteristic of the hallux $(\mathrm{M})$, the forefoot $(\mathrm{N})$, the arch $(\mathrm{P})$ and the heel $(\mathrm{Q})$. According to the gait characteristics of the people, the phase labels of the gait data is obtained through the collected pressure signal. Figure 4 shows a snapshot of the signal acquisition process, and ABCDE represents five gaits during the subject motion. The selected gait characteristics are shown in Table 1, and the gait phase is classified, as shown in Figure 5:

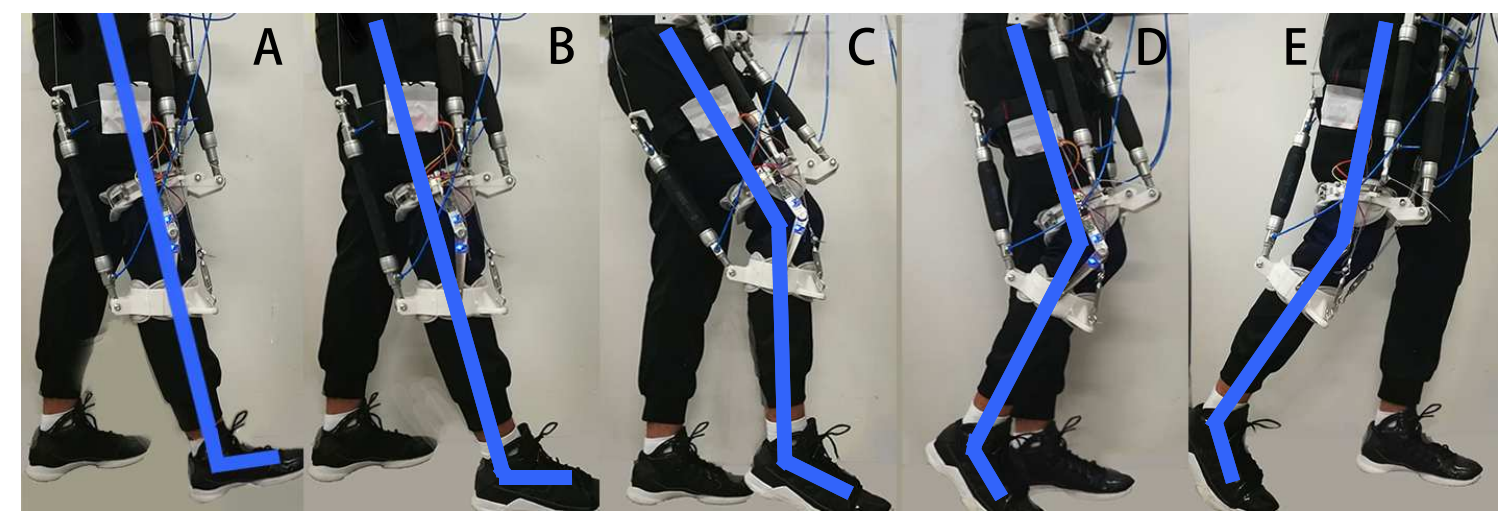

Figure 4. The subject motion snapshot 
Table 1. Gait characteristics correspondence table in each state. (1 means the force is gradually increasing, and -1 means the force is gradually decreasing. 0 means the position is not under pressure.)

\begin{tabular}{ccccccccc}
\hline \multirow{2}{*}{ Pressure Feature } & \multicolumn{3}{c}{ Plantar Pressure } & \multicolumn{4}{c}{ Plantar Pressure Trend } \\
\cline { 2 - 9 } & $S_{Q}$ & $S_{\boldsymbol{P}}$ & $\boldsymbol{S}_{\boldsymbol{N}}$ & $\boldsymbol{R}_{\boldsymbol{M}}$ & $\boldsymbol{R}_{\boldsymbol{Q}}$ & $\boldsymbol{R}_{\boldsymbol{P}}$ & $\boldsymbol{R}_{\boldsymbol{N}}$ & $\boldsymbol{S}_{\boldsymbol{M}}$ \\
\hline (A) init-st & 1 & 0 & 0 & 0 & 1 & 1 and -1 & 1 and -1 & 1 and -1 \\
(B) mid-st & 1 & 1 & 1 & 1 & 1 and -1 & 1 and -1 & 1 & 1 \\
(C) term-st & 0 & 1 & 1 & 1 & 1 and -1 & -1 & 1 and -1 & 1 \\
(D) pre-st & 0 & 0 & 1 & 1 & 1 and -1 & 1 and -1 & -1 & 1 and -1 \\
(E) swing phase & 0 & 0 & 0 & 0 & 1 and -1 & 1 and -1 & 1 and -1 & 1 and -1 \\
\hline
\end{tabular}

The real-time phase is identified, so the gait can be predicted in advance: this work benefits from smooth switching of the controller. First of all, the gait should be classified. The classification result of gait phase is shown in Figure 5.

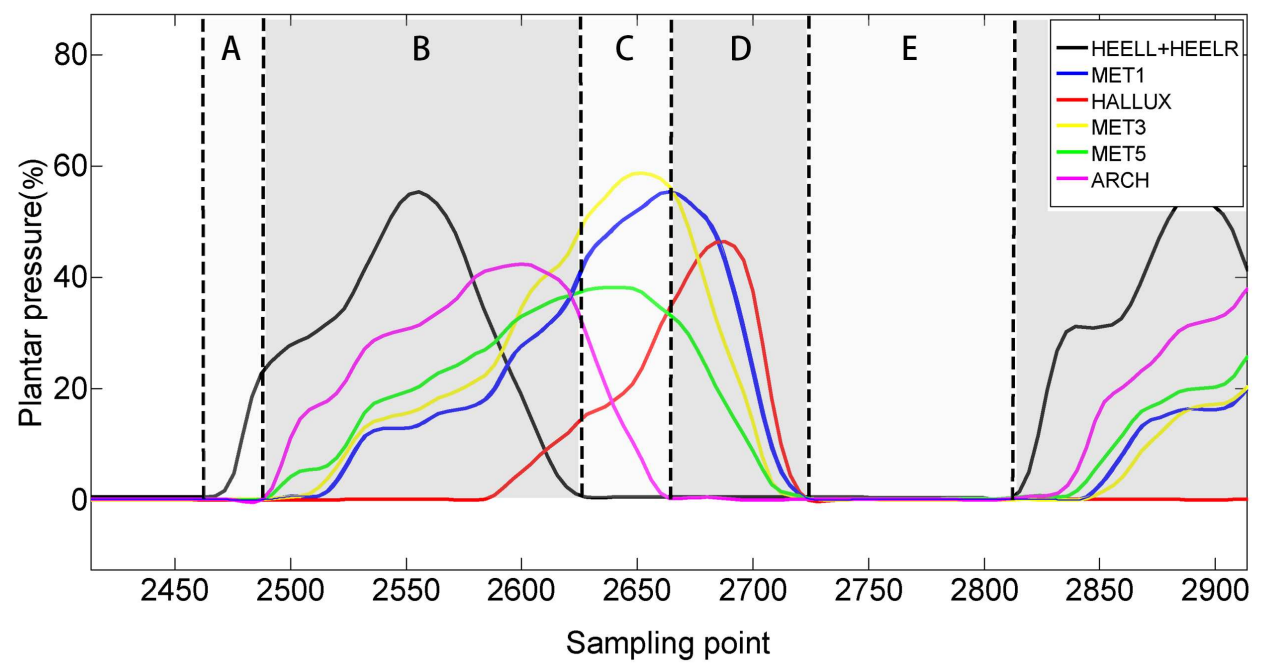

Figure 5. Gait phase classified by plantar pressure data. This picture shows how the pressure data of one cycle can be divided into five gaits. The starting point of the black line represents the touch of the ground by the heel; the starting point of the purple line represents the full touch of the right foot (left toe off the ground); the end of the black line represents the right heel off the ground (the left and right feet are exchanged); the end of the purple line represents the right arch of the foot is off the ground (the left heel touches the ground); the end of the red line represents the right toe off the ground.

As a neural network, the Extreme Learning Machine can be applied to the train the phase detector and complete the gait recognition, which is designed by the following specific steps:

1. The number of neurons in the hidden layer is 200;

2. The activation function of the hidden layer neurons is $\operatorname{sigmoid}(x)=\left(\frac{1}{1+e^{-x}}\right)$, and computes the hidden layer output matrix $H$;

3. Compute output layer weight $\hat{\beta}: \hat{\beta}=H+T^{\prime}$.

The extreme learning machine model based on the plantar pressure signal has good prediction accuracy. Figure 6 shows the classification result of data from 1000 gaits (actual test sets). Experiments show that the extreme learning machine can guarantee the accuracy to more than $95 \%$, so it has practical meaning to use this identification model in the switching motion of the exoskeleton. 


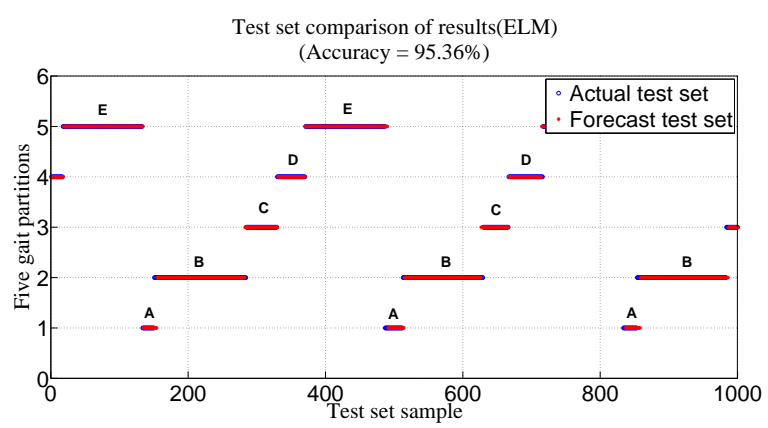

Figure 6. Gait phase classified by plantar pressure datas. Stages A, B, C, D, and E correspond to the stages divided in Figure 5. ABCD are the states of the stance phase; $\mathrm{E}$ is the state of the swing phase. The heel touches the ground only in stage A, and the hallux touches only in stage D, so A is considered to be the phase pre-conversion stage from the swing phase to the stance phase, and $D$ is the phase pre-conversion stage from the stance phase to the swing phase. The phase smoothing switching strategy is performed in these two stages, which is described later.

\subsubsection{Smoothing Switch of Control Torque}

Pneumatic artificial muscles differ from conventional motors, because pneumatic artificial muscles need take a short time to contract and relax. During the gait phase transition, the models under different phases produce changes in the model parameters and cause discontinuous control input torque. To avoid the sudden change of the controller, a smoothing method is introduced. This method is valid in the pre-conversion stage. $f_{\text {weight }}$ is weight function whose amplitude is 1 , as shown in Figure 7. The sampling interval $\mathrm{N}$ of the weight function corresponds to the actual gait switching interval time, which is selected as $50-100 \mathrm{~ms}$. $\tau_{\text {smoothing }}$ is used to replace $\tau_{\text {original }}$ within the switching interval. Smoothing method performance is shown in Figure 8.

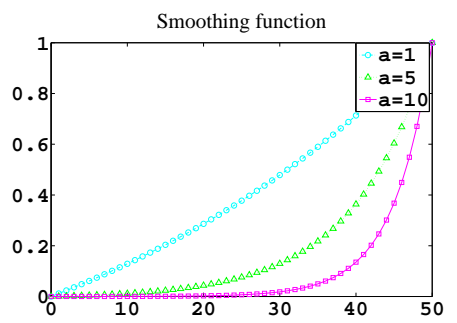

Weight function
$f_{\text {weight }}=\left(e^{a \times\left(t-t_{i}\right) / N}-1\right) /\left(e^{a}-1\right)$
$\tau_{\text {original }} \times f_{\text {weighting }}$, if $t \in\left(t_{i}, t\right)$
1, otherwise

Figure 7. Gradual smooth curve.

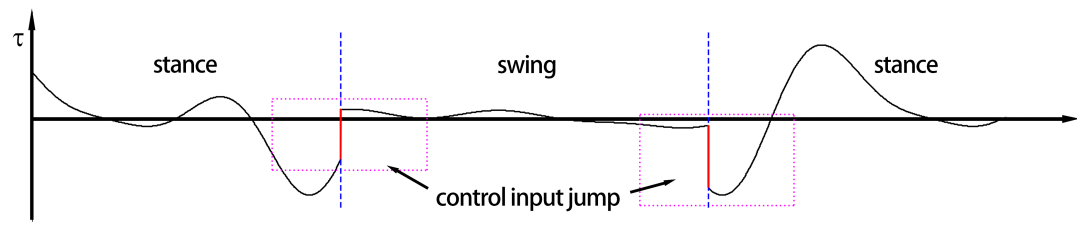

(a)

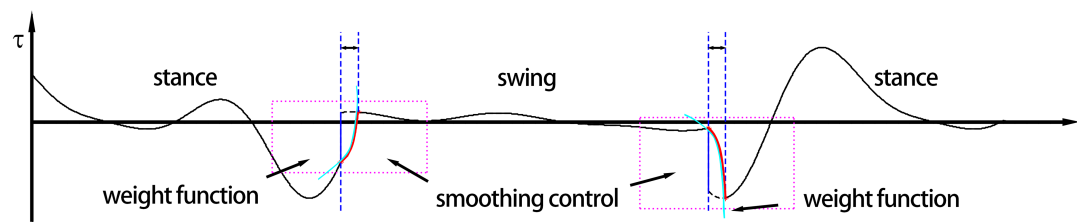

(b)

Figure 8. The effect of the smoothing method in the pre-conversion process: (a) shows the control input jump when switching; (b) avoids the sudden-jump of the control input by smoothing method, this has practical application value for pneumatic muscle. 


\section{Experimental Results and Discussion}

\subsection{Selection of Parameters}

To verify the performance of the closed-loop system as shown in Figure 1, the following simulation experiments are performed. Assuming that the average height of males as $l=1.75 \mathrm{~m}$ and the average weight as $m=65 \mathrm{~kg}$, according to the data published in [29,30], the corresponding human-body parameters can be obtained. The parameters of the lower extremity exoskeleton system are shown in Table 2.

Table 2. Exoskeleton system main parameters.

\begin{tabular}{cccc}
\hline Number & $m_{i} /(\mathbf{k g})$ & $l_{i} /(\mathrm{m})$ & $r_{i} /(\mathrm{m})$ \\
\hline 1 & 9.933 & 0.1 & 0.117 \\
2 & 2.269 & 0.1 & 0.352 \\
3 & 1 & 0.469 & 0.093 \\
4 & 1 & 0.372 & 0.2 \\
5 & 1 & 0.05 & 0.15 \\
6 & 29.378 & - & - \\
\hline
\end{tabular}

\subsection{Simulation and Comparison Experments}

In this section, the simulation experiment is implemented, and the performance of the proposed control algorithm is studied by simulation platform. The following primary analysis is completed: First, this work verifies the effectiveness of the sliding mode control method combined with the extended state observer for the lower extremity exoskeleton. Secondly, the smoothing method solves the time requirement concerning the pneumatic muscle contraction and relaxation. Thirdly, the result shows that the motion characteristic of pneumatic artificial muscles meets the control requirement for the exoskeleton movement. Add un-modelled uncertainties to the nominal model parameters, and the total uncertain disturbances are related to system information and selected as $d=m \operatorname{sgn}(q)+n q$, where $m=\operatorname{diag}(2,2), n=\operatorname{diag}(0.2,0.2)$. The desired trajectory of lower extremity exoskeleton in the gait cycle are obtained by VICON three-dimensional gait analysis system:

$$
q_{d}=\left\{\begin{array}{l}
q_{\text {hip }}=0.017(82.24-21.38 \cos (5.06 t)+8.347 \sin (5.06 t)+5.319 \cos (10.12 t) \\
-3.161 \cos (10.12 t)-0.535 \cos (15.18 t)-2.624 \sin (15.18 t)) \\
q_{k n e e}=0.017(25.08-7.049 \cos (5.18 t)-21.46 \sin (5.18 t)-16.29 \cos (10.39 t) \\
+13.220 \sin (10.39 t)+1.332 \cos (15.55 t)+4.175 \sin (15.55 t))
\end{array}\right.
$$

The system initial state $x_{0}=\left[\begin{array}{llll}1.2 & 0.15 & -0.5 & 0.5\end{array}\right]$. The swing phase and the stance phase controller gains are $k_{g s w}=\left[\begin{array}{cc}100 & 0 \\ 0 & 100\end{array}\right], k_{g s t}=\left[\begin{array}{cc}20 & 0 \\ 0 & 20\end{array}\right]$ respectively. The conventional controller (16) sets $\eta=\left[\begin{array}{ll}5 & 0 \\ 0 & 5\end{array}\right]$, while the new one(17) can be set as $\eta=\left[\begin{array}{cc}0.1 & 0 \\ 0 & 0.1\end{array}\right]$. To avoid peaking phenomenon, the initial value of the ESO is set to be the same as the initial value of the system states and disturbances. To ensure the stability of the system, $\bar{A}$ in Equation (8) need to be guaranteed for Hurwitz, that is

$$
\alpha_{1}=\left[\begin{array}{ll}
6 & 0 \\
0 & 6
\end{array}\right], \alpha_{2}=\left[\begin{array}{cc}
10 & 0 \\
0 & 10
\end{array}\right], \alpha_{3}=\left[\begin{array}{ll}
6 & 0 \\
0 & 6
\end{array}\right]
$$

In the actual test of the lower extremity exoskeleton motion, the phase switching time is determined by the phase detector we mention ahead. Assuming there are twice switching in $2 \mathrm{~s}$, and gait switching signal is shown in Figure 9. 


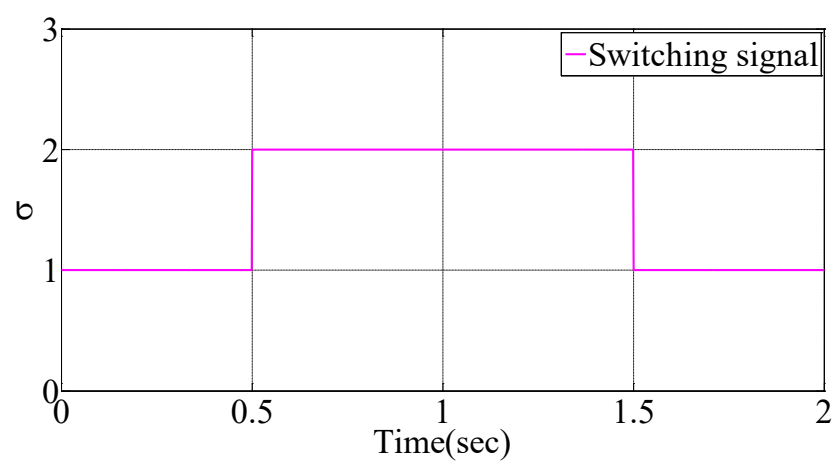

Figure 9. This is a switching signal that triggers twice switching within $2 \mathrm{~s}$, at $0.5 \mathrm{~s}$ and $1.5 \mathrm{~s}$ respectively. $\sigma$ denotes the phase of the exoskeleton. (value 1 denotes the swing phase and 2 denotes stance phase.)

The simulation result can be found in Figures 10-15. The tracking control performance of Hybrid Phase Sliding Mode Control Scheme is shown in Figures 10 and 11, where Figure 10 is the angle tracking curve and Figure 11 is the angular velocity tracking curve of the double joints. The tracking error between "ESO+SMC" and desired trajectory is markedly shown in Figure 12. During the gait phase switching, although the tracking error increases, it is small enough to meet the control requirement. Therefore, the results show that the controller, together with an extended state observer, has excellent control performance for the exoskeleton system. Figure 13 shows that the extended state observer has good observation ability for strong disturbances. It can be seen that the exoskeleton system by this method can adapt to a relatively bad environment and still obtain good control performance.
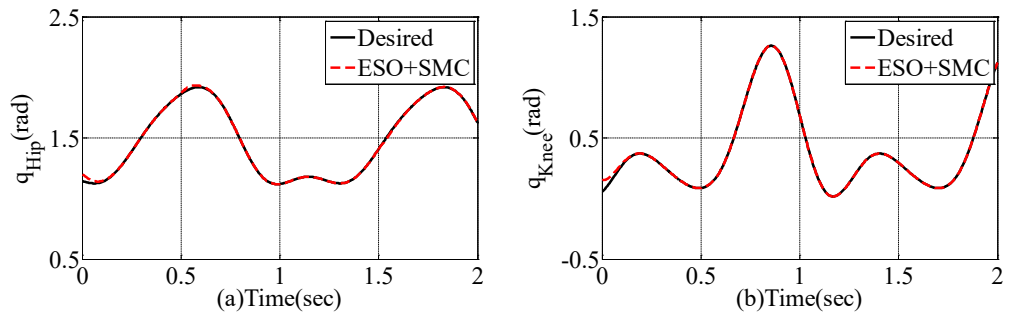

Figure 10. Joint angle tracking of the hybrid phase: (a) Hip joint; (b) Knee joint.

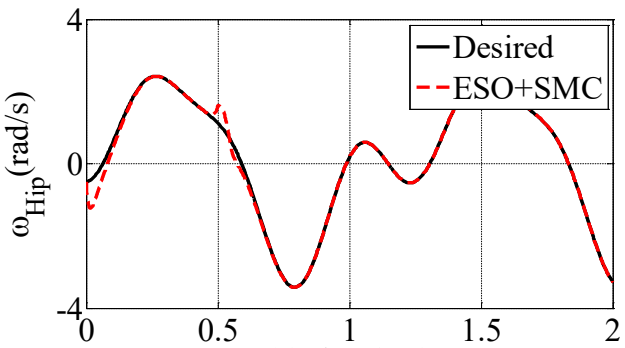

(a)Time(sec)

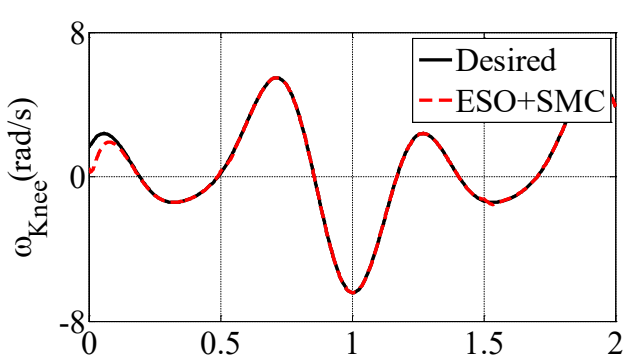

(b)Time(sec)

Figure 11. Joint angle velocity tracking of the hybrid phase: (a) Hip joint; (b) Knee joint.

The control inputs of the traditional SMC controller (16) and ESO+SMC (17) are shown in Figure 14. The traditional one shows the great effect of chattering while the other eliminates this phenomenon. The control torques combining with smoothing method and without are shown in Figure 15. There is an apparent jump in the stage of gait switching without smoothing method, while the smoothing method turns this jump into a slower change. 

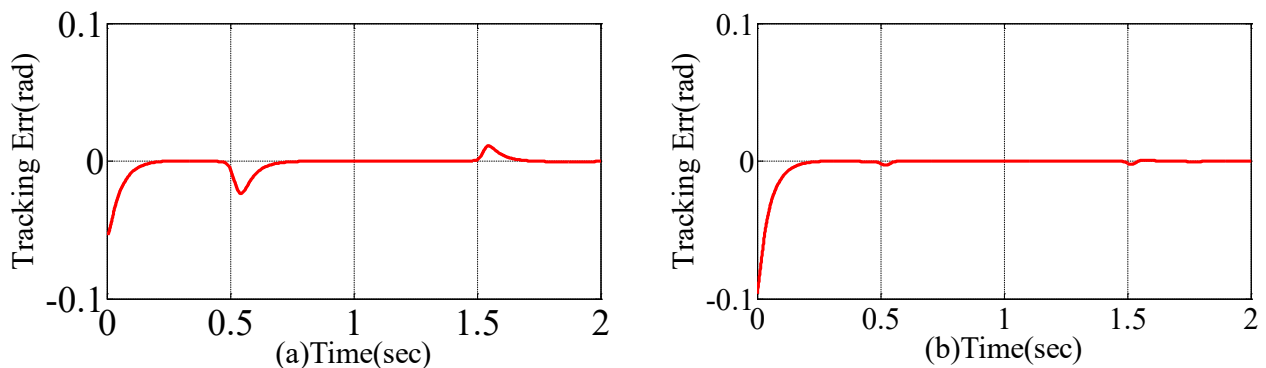

Figure 12. Joint angle tracking error: (a) Hip joint; (b) Knee joint.

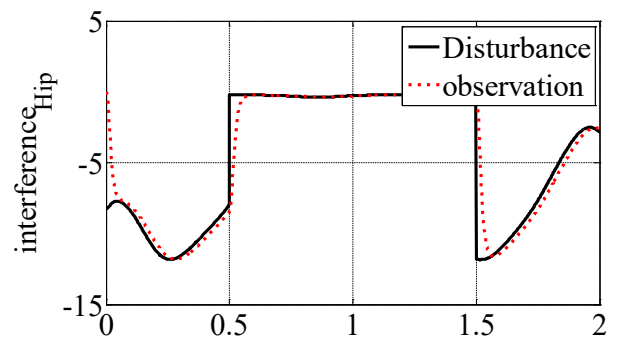

(a)Time $(\mathrm{sec})$

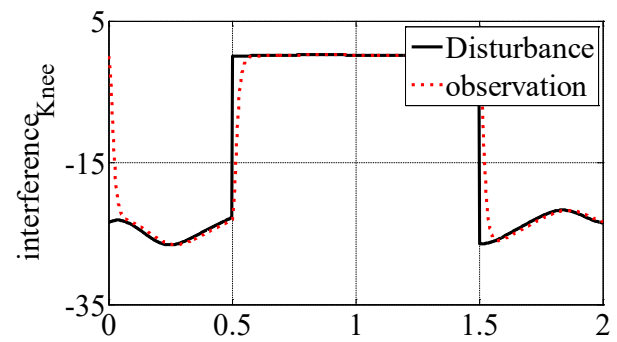

(b) Time(sec)

Figure 13. Observation curve of total disturbances: (a) Hip joint; (b) Knee joint.
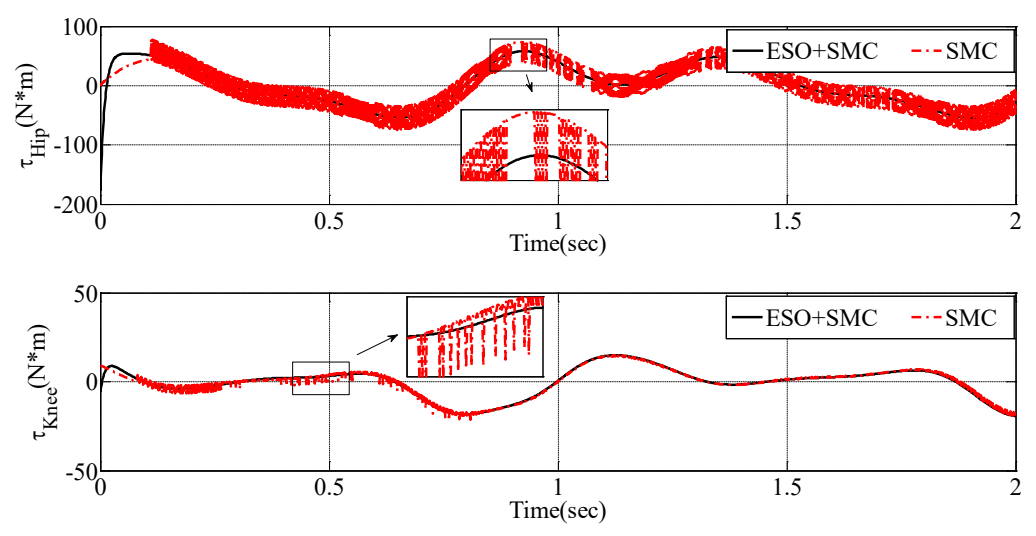

Figure 14. Comparison of sliding mode controllers.
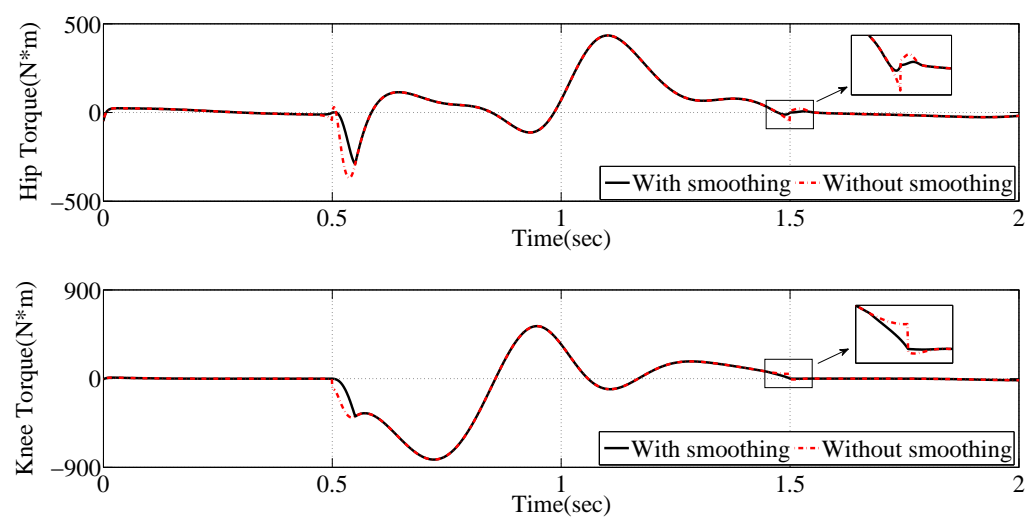

Figure 15. Smoothing control input curve. 
According to the schematic diagram of the lower extremity exoskeleton in the sagittal plane, the structural angle and the shrinkage of the pneumatic muscle under ideal conditions can be analyzed easily by the Cosine theorem (See Appendix B for details). Figure 16 shows the shrinkage percentage of the artificial pneumatic muscles under smoothing input. When the contraction rate of pneumatic muscle arrives about $25 \%$ or less than $25 \%$, the action range of pneumatic muscle is considered reasonable according to FESTO pneumatic muscle product manual. Pneumatic muscle 2 and pneumatic muscle 3 simulate the antagonistic muscles on the upper and lower sides of the knee joint. Thus, the contraction rate curve of the pneumatic muscle 2 and the pneumatic muscle 3 are approximately opposite. However, the changing trends of them are analogous in the shadow A and B. The main reason is that pneumatic muscle 2 may cross the knee joint when the knee joint rotates in the direction of increasing knee joint angle.

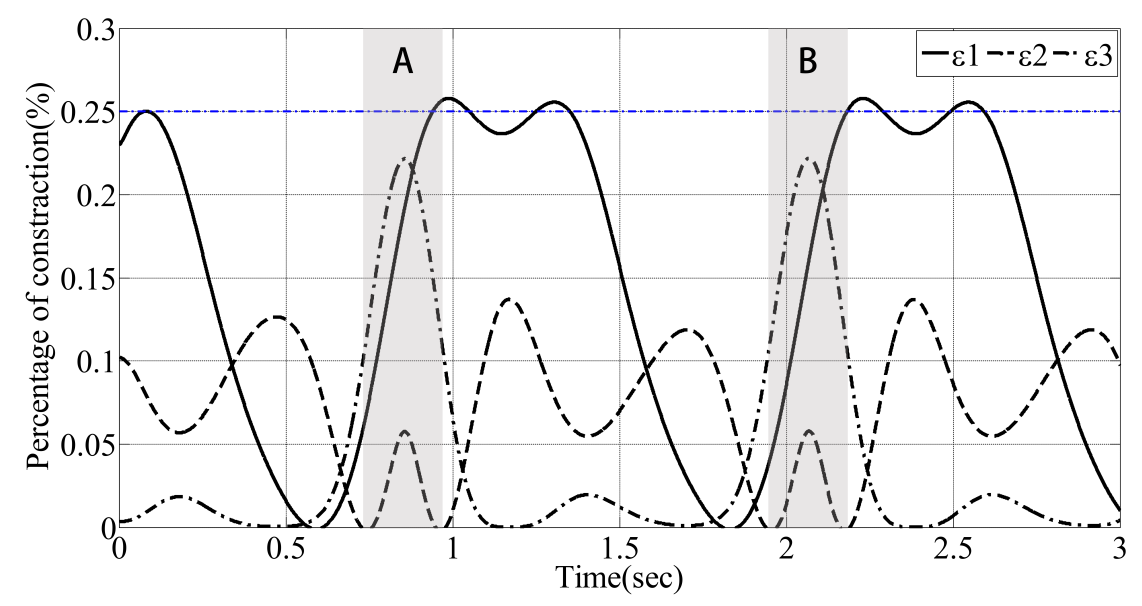

Figure 16. The trajectory of three pneumatic muscles during the assisting process.

\section{Conclusions}

In this article, a hybrid phase sliding mode control scheme is proposed to control double joint lower extremity exoskeleton, which is driven by artificial pneumatic muscle. First, the lower extremity exoskeleton phase models are established by the Euler-Lagrange method, respectively. The extended state observer and sliding mode controller in hybrid phase are designed together. This robust control method can adapt to the nonlinear and strong coupling characteristics of the model and provide excellent observation ability for internal or external disturbances. Secondly, the phase detector designed by the Extreme Learning Machine has strong gait-classification and phase-detection capability through offline learning, and then it helps the system adjust the corresponding controller by online identification. According to the phase detector, the controller and the plant switch at the same time. Finally, the smoothing method plays a role in avoiding the sudden-jump of the controller during the pre-conversion stage, which has practical significance for the exoskeleton. The simulation experiment proves that this method provides excellent control performance for the exoskeleton. Moreover, the contraction rate curve of the artificial pneumatic muscles verifies that the installation position of the pneumatic muscle $1-3$ is reasonable.

The major contribution of this study has great practical significance for lower extremity exoskeleton driven by pneumatic muscles, so this mechanism will be applied to the lower extremity exoskeleton to verify safety and comfort in the future.

Author Contributions: Resources, C.W.; Supervision, L.C. and J.W.; Validation, L.C., C.W., J.W. and X.S.; Writing-original draft, C.W.; Writing-review \& editing, L.C., C.W. and J.W.

Funding: This research was funded by National Natural Science Foundation of China $(61703135,61703134)$, Natural Science Foundation of Hebei Province (F2017202119), and 2019 Graduate Innovation Funding Project of Hebei Province (220056).

Conflicts of Interest: The authors declare no conflict of interest. 


\section{Appendix A. Dynamic Model}

The specific form of the coefficient matrix in Equation (1) is given below: the inertia matrix is,

$$
F(q)=\left[\begin{array}{ll}
F_{11} & F_{12} \\
F_{21} & F_{22}
\end{array}\right]
$$

while $i=1$, corresponding to the model of swing phase:

$$
\begin{aligned}
F_{11}= & 0.583 * m_{1} * l_{3}^{2}+0.5 * m_{2} *\left(1.1667 * l_{4}^{2}+2 * l_{3}^{2}+2 * l_{3} * l_{4} * \cos \left(2 * q_{1}+q_{2}\right)\right) \\
& +0.5 * m_{3} *\left(0.5 * r_{4}^{2}+0.5 * l_{3}^{2}+r_{4} * l_{3} * \cos \left(2 * q_{1}+q_{2}\right)\right)+0.5 * m_{4} * r_{2}^{2}+0.5 * m_{5} * \\
& \left(0.5 * r_{3}^{2}+0.5 * l_{5}^{2}+\left(r_{1}-l_{3}\right)^{2}-\left(r_{1}-l_{3}\right) * r_{3} * \cos \left(2 * q_{1}+q_{2}\right)-\left(r_{1}-l_{3}\right) * l_{5} * \sin \left(q_{2}\right)\right) \\
F_{12}= & F_{21}=0.5 * m_{2} *\left(1.1667 * l_{4}^{2}+l_{3} * l_{4} * \cos \left(2 * q_{1}+q_{2}\right)\right) \\
& +0.5 * m_{3} *\left(0.5 * r_{4}^{2}+r_{4} * l_{3} * \cos \left(2 * q_{1}+q_{2}\right)\right)+0.5 * m_{5} * \\
& \left(0.5 * r_{3}^{2}+0.5 * l_{5}^{2}-0.5 *\left(r_{1}-l_{3}\right) * r_{3} * \cos \left(2 * q_{1}+q_{2}\right)-0.5 *\left(r_{1}-l_{3}\right) * l_{5} * \sin \left(q_{2}\right)\right) \\
F_{22}= & 0.5833 * m_{2} * l_{4}^{2}+0.25 * m_{3} * r_{4}^{2}+0.25 * m_{5} * r_{3}^{2}+0.25 * m_{5} * l_{5}^{2}
\end{aligned}
$$

while $i=2$, corresponding to the model of stance phase:

$$
\begin{aligned}
& F_{11}=\left(1 / 3 * m_{2} * l_{2}^{2}+1 / 3 * m_{1} * l_{1}^{2}+m_{1} * l_{2}^{2}+0.25 * m_{1} * l_{1}^{2}+m_{1} * l_{1} * l_{2} * \cos \left(q_{2}\right)+m_{6} * l_{1}^{2}\right. \\
& +m_{6} * l_{2}^{2}+m_{6} * l_{1} * l_{2} * \cos \left(q_{2}\right)+m_{3} * l_{2}^{2}+m_{6} *\left(l_{1}-0.5 * r_{2}\right)^{2}+m_{3} * l_{2} *\left(l_{1}-0.5 * r_{2}\right) \\
& * \cos \left(q_{2}\right)+m_{4} *\left(l_{5}^{2} * \cos \left(q_{1}+q_{2}\right)^{2} /\left(4 * \sin \left(q_{1}+q_{2}\right)^{2}\right)\right)+l_{5} *\left(l_{2}-0.5 * r_{2}\right) \\
& * \cos \left(q_{1}+q_{2}\right) / \sin \left(q_{1}+q_{2}\right)+\left(l_{2}-0.5 * r_{2}\right)^{2} * \sin \left(q_{1}+q_{2}\right)^{2}+\left(l_{1}-0.5 * r_{3}-0.5 * l_{5}\right)^{2} \\
& * \cos \left(q_{1}+q_{2}\right)^{2}+l_{5} *\left(l_{1}-r_{1}\right) * \cos \left(q_{1}+q_{2}\right) / 2 / \sin \left(q_{1}+q_{2}\right)^{2}+\left(l_{1}-r_{1}\right) *\left(l_{2}-0.5 * r_{2}\right) \\
& \left.* \sin \left(q_{1}\right) * \sin \left(q_{1}+q_{2}\right)+\left(l_{2}-r_{1}\right) *\left(l_{2}-0.5 * r_{3}-0.5 * r_{5}\right) * \cos \left(q_{1}+q_{2}\right)+0.25 *\left(l_{1}-r_{1}\right)^{2}\right) \\
& +m_{5} *\left(l_{2}-0.5 * r_{4}\right)+0.25 * l_{1}^{2} * \cos \left(q_{1}\right)+m_{5} * l_{1} *\left(l_{1}-0.5 * r_{4}\right) * \cos \left(q_{1}\right) * \cos \left(q_{1}+q_{2}\right) \\
& F_{12}=F_{21}=\left(m_{1} * l_{1}^{2}+0.5 * m_{1} * l_{1} * l_{2} * \cos \left(q_{2}\right)+m_{6} * l_{2}^{2}+m_{6} * l_{1} * l_{2} * \cos \left(q_{2}\right)+m_{3} * l_{2}^{2}\right. \\
& +m_{3} * l_{2} *\left(l_{1}-0.5 * r_{2}\right) * \cos \left(q_{2}\right)+m_{4} *\left(l_{5}^{2} * \cos \left(q_{1}+q_{2}\right)^{2} /\left(4 * \sin \left(q_{1}+q_{2}\right)^{4}\right)\right. \\
& +l_{5} *\left(l_{2}-0.5 * r_{2}\right) * \cos \left(q_{1}+q_{2}\right) / \sin \left(q_{1}+q_{2}\right)+\left(l_{2}-0.5 * r_{2}\right)^{2} * \sin \left(q_{1}+q_{2}\right)^{2} \\
& \left.+\left(l_{1}-0.5 * r_{3}-0.5 * l_{5}\right)^{2} * \cos \left(q_{1}+q_{2}\right)^{2}\right)+0.5 * m_{4} *\left(l_{5} *\left(l_{1}-0.5 * r_{1}\right)\right. \\
& * \cos \left(q_{1}+q_{2}\right) / 2 / \sin \left(q_{1}+q_{2}\right)^{2}+\left(l_{1}-r_{1}\right) *\left(l_{2}-0.5 * r_{2}\right) * \sin \left(q_{2}\right) \\
& \left.* \sin \left(q_{1}+q_{2}\right)+\left(l_{2}-r_{1}\right) *\left(l_{2}-0.5 * r_{3}-0.5 * r_{5}\right) * \cos \left(q_{1}\right) * \cos \left(q_{1}+q_{2}\right)\right) \\
& +m_{5} *\left(l_{3}-0.5 * r_{4}\right)^{2}+0.5 * m_{5} * l_{1} *\left(l_{2}-0.5 * r_{2}\right) * \cos \left(q_{1}\right) * \cos \left(q_{1}+q_{2}\right) \\
& F_{22}=\left(1 / 3 * m_{2} * l_{2}^{2}+m_{1} * l_{2}^{2}+m_{6} * l_{2}^{2}+m_{5} *\left(l_{2}-0.5 * r_{4}\right)^{2}+m_{4} *\left(l_{5}^{2} * \cos \left(q_{1}+q_{2}\right)^{2}\right.\right. \\
& /\left(4 * \sin \left(q_{1}+q_{2}\right)^{4}\right)+l_{5} *\left(l_{2}-0.5 * r_{2}\right) * \cos \left(q_{1}+q_{2}\right) / \sin \left(q_{1}+q_{2}\right)+\left(l_{2}-0.5 * r_{2}\right)^{2} \\
& * \sin \left(q_{1}+q_{2}\right)^{2}+\left(l_{2}-0.5 * r_{3}-0.5 * l_{5}\right)^{2} * \cos \left(q_{1}+q_{2}\right)^{2}
\end{aligned}
$$

the centripetal and Coriolis matrix is,

$$
H(q, \dot{q})=\left[\begin{array}{ll}
H_{11} & H_{12} \\
H_{21} & H_{22}
\end{array}\right]
$$

while $i=1$, corresponding to the model of swing phase:

$$
\begin{aligned}
H_{11} & =\left(-m_{2} * l_{3} * l_{4} * \sin \left(2 * q_{1}+q_{2}\right)-0.5 * m_{3} * r_{4} * l_{3} * \sin \left(2 * q_{1}+q_{2}\right)\right) * d q_{1} \\
& +\left(0.75 * m_{5} * r_{3} *\left(r_{1}-l_{3}\right) * \sin \left(2 * q_{1}+q_{2}\right)-0.25 * m_{5} * r_{3} *\left(r_{1}-l_{3}\right) * \sin \left(q_{2}\right)\right) * d q_{1} \\
H_{12}= & \left(-0.5 * m_{2} * l_{3} * l_{4} * \sin \left(2 * q_{1}+q_{2}\right)-0.25 * m_{3} * r_{4} * l_{3} * \sin \left(2 * q_{1}+q_{2}\right)\right) * d q_{2} \\
& +\left(0.5 * m_{5} *\left(r_{1}-l_{3}\right) * r_{3} * \sin \left(2 * q_{1}+q_{2}\right)+0.5 * m_{5} *\left(r_{1}-l_{3}\right) * l_{5} * \cos \left(2 * q_{1}+q_{2}\right)\right) * d q_{2} \\
& +\left(-m_{2} * l_{3} * l_{4} * \sin \left(2 * q_{1}+q_{2}\right)-0.5 * m_{5} *\left(r_{1}-l_{3}\right) * l_{5} * \cos \left(2 * q_{1}+q_{2}\right)\right) * d q_{1} \\
& +\left(-0.25 * m_{5} *\left(r_{1}-l_{3}\right) * r_{3} * \sin \left(2 * q_{1}+q_{2}\right)-0.25 * m_{5} *\left(r_{1}-l_{3}\right) * l_{5} * \sin \left(q_{2}\right)\right) * d q_{1} \\
& +\left(-m_{3} * r_{4} * l_{3} * \sin \left(2 * q_{1}+q_{2}\right)\right) * d q_{1}
\end{aligned}
$$




$$
\begin{aligned}
H_{21} & =\left(0.25 * m_{5} *\left(r_{1}-l_{3}\right) * r_{3} * \sin \left(2 * q_{1}+q_{2}\right)+0.5 * m_{2} * l_{3} * l_{4} * \sin \left(2 * q_{1}+q_{2}\right)\right) * d q_{1} \\
& +\left(0.25 * m_{3} * r_{4} * l_{3} * \sin \left(2 * q_{1}+q_{2}\right)+0.5 * m_{5} *\left(r_{1}-l_{3}\right) * l_{5} * \cos \left(2 * q_{1}+q_{2}\right)\right) * d q_{1} \\
& +\left(-m_{2} * l_{3} * l_{4} * \sin \left(2 * q_{1}+q_{2}\right)+0.25 * m_{3} * r_{4} * l_{3} * \sin \left(q_{1}\right)\right) * d q_{2} \\
& +\left(-0.25 * m_{5} *\left(r_{1}-l_{3}\right) * l_{5} * \cos \left(q_{2}\right)+0.25 * m_{5} *\left(r_{1}-l_{3}\right) * l_{5} * \cos \left(2 * q_{1}+q_{2}\right)\right) * d q_{2} \\
& +\left(0.25 * m_{3} * r_{4} * l_{3} * \sin \left(2 * q_{1}+q_{2}\right)\right) * d q_{2} \\
H_{22} & =0
\end{aligned}
$$

while $i=2$, corresponding to the model of stance phase:

$$
\begin{aligned}
& H_{11}=\left(\left(m _ { 4 } * \left(0 . 2 5 * l _ { 5 } ^ { 2 } * \left(2 * \cos \left(q_{1}+q_{2}\right) / \sin \left(q_{1}+q_{2}\right)^{3}+4 * \cos \left(q_{1}+q_{2}\right) / \sin \left(q_{1}+q_{2}\right)^{5}\right.\right.\right.\right. \\
& +l_{5} *\left(l_{2}-0.5 * r_{2}\right) / \sin \left(q_{1}+q_{2}\right)^{2}+2 *\left(l_{2}-0.5 * r_{2}\right)^{2} * \sin \left(q_{1}+q_{2}\right) * \cos \left(q_{1}+q_{2}\right) \\
& -\left(l_{1}-0.5 * r_{3}-0.5 * l_{5}\right)^{2} * \sin \left(q_{1}+q_{2}\right)+l_{5} *\left(l_{1}-r_{1}\right) / 2 *\left(1 / \sin \left(q_{1}+q_{2}\right)\right. \\
& +2 * \cos \left(q_{1}+q_{2}\right)^{2} / \sin \left(q_{1}+q_{2}\right)^{3}+\left(l_{1}-r_{1}\right) *\left(l_{2}-0.5 * r_{2}\right) *\left(\sin \left(q_{1}\right) * \cos \left(q_{1}+q_{2}\right)\right) \\
& \left.+\left(l_{2}-r_{1}\right) *\left(l_{2}-0.5 * r_{3}-0.5 * r_{5}\right) *\left(\cos \left(q_{1}\right) * \sin \left(q_{1}+q_{2}\right)\right)\right)-m_{5} * l_{1} *\left(l_{1}-0.5 * r_{4}\right) \\
& \left.\left.* \cos \left(q_{1}\right) * \sin \left(q_{1}+q_{2}\right)\right) * d q_{2}\right)+\left(m_{1} * g * l_{2} * \cos \left(q_{1}+q_{2}\right)+0.5 * m_{1} * g * l_{1} * \cos \left(q_{1}\right)\right. \\
& +0.5 * m_{2} * g * l_{2} * \cos \left(q_{1}+q_{2}\right)+m_{6} * g * l_{1} * \cos \left(q_{1}\right)+m_{3} * g * l_{2} * \cos \left(q_{1}+q_{2}\right) \\
& +m_{3} * g *\left(l_{1}-0.5 * r_{2}\right) * \cos \left(q_{1}\right)+m_{4} * g *\left(l_{1}-0.5 * r_{3}-0.5 * l_{5}\right) * \cos \left(q_{1}+q_{2}\right) \\
& +0.5 * m_{4} * g *\left(l_{2}-r_{1}\right) * \cos \left(q_{1}\right)+m_{5} * g *\left(l_{2}-0.5 * r_{4}\right) * \cos \left(q_{1}+q_{2}\right) \\
& \left.+0.5 * m_{5} * g * l_{1} * \cos \left(q_{1}\right)\right) \\
& H_{12}=\left(\left(-0.5 * m_{1} * l_{1} * l_{2} * \sin \left(q_{2}\right)-m_{6} * l_{1} * l_{2} * \sin \left(q_{2}\right)-m_{3} * l_{2} *\left(l_{1}-0.5 * r_{2}\right) * \sin \left(q_{2}\right)\right.\right. \\
& +m_{4} *\left(0.25 * l_{5}^{2} *\left(2 * \cos \left(q_{1}+q_{2}\right) / \sin \left(q_{1}+q_{2}\right)^{3}+2 * \cos \left(q_{1}+q_{2}\right)^{3} / \sin \left(q_{1}+q_{2}\right)^{3}\right)\right. \\
& +l_{5} *\left(l_{2}-0.5 * r_{2}\right) / \sin \left(q_{1}+q_{2}\right)^{2}+2 *\left(l_{2}-0.5 * r_{2}\right)^{2} * \sin \left(q_{1}+q_{2}\right) * \cos \left(q_{1}+q_{2}\right) \\
& -2 *\left(l_{1}-0.5 * r_{3}-0.5 * l_{5}\right)^{2} * \sin \left(q_{1}+q_{2}\right) * \cos \left(q_{1}+q_{2}\right)+l_{5} *\left(l_{1}-0.5 * r_{1}\right) / 4 \\
& /\left(1 / \sin \left(q_{1}+q_{2}\right)+2 * \cos \left(q_{1}+q_{2}\right)^{2} / \sin \left(q_{1}+q_{2}\right)^{3}\right)+0.5 *\left(l_{1}-r_{1}\right) *\left(l_{2}-0.5 * r_{2}\right) \\
& * \sin \left(q_{1}\right) * \cos \left(q_{1}+q_{2}\right)-0.5 *\left(l_{2}-r_{1}\right) *\left(l_{2}-0.5 * r_{3}-0.5 * r_{5}\right) * \cos \left(q_{1}\right) * \cos \left(q_{1}+q_{2}\right) \\
& \left.\left.-0.5 * m_{5} * l_{1} *\left(l_{1}-0.5 * r_{4}\right) * \cos \left(q_{1}\right) * \sin \left(q_{1}+q_{2}\right)\right) * d q_{2}\right) \\
& H_{21}=\left(m_{4} * 0.25 * l_{5}^{2} *\left(2 * \cos \left(q_{1}+q_{2}\right) / \sin \left(q_{1}+q_{2}\right)^{3}+2 * \cos \left(q_{1}+q_{2}\right)^{3} / \sin \left(q_{1}+q_{2}\right)^{3}\right)\right. \\
& +l_{5} *\left(l_{2}-0.5 * r_{2}\right) / \sin \left(q_{1}+q_{2}\right)^{2}+2 *\left(l_{2}-0.5 * r_{2}\right)^{2} * \sin \left(q_{1}+q_{2}\right) * \cos \left(q_{1}+q_{2}\right) \\
& -2 *\left(l_{1}-0.5 * r_{3}-0.5 * l_{5}\right)^{2} * \sin \left(q_{1}+q_{2}\right) * \cos \left(q_{1}+q_{2}\right)+l_{5} *\left(l_{1}-r_{1}\right) / 4 \\
& *\left(1 / \sin \left(q_{1}+q_{2}+2 * \cos \left(q_{1}+q_{2}\right)^{2} / \sin \left(q_{1}+q_{2}\right)^{3}+0.5 *\left(l_{1}-r_{1}\right) *\left(l_{2}-0.5 * r_{2}\right)\right.\right. \\
& \left.* \sin \left(q_{1}\right) * \cos \left(q_{1}+q_{2}\right)+0.5 *\left(l_{2}-r_{1}\right) *\left(l_{2}-0.5 * r_{3}-0.5 * r_{5}\right) * \cos \left(q_{1}\right) * \sin \left(q_{1}+q_{2}\right)\right) \\
& \left.+0.5 * m_{5} * l_{1} *\left(l_{1}-0.5 * r_{4}\right) * \cos \left(q_{1}\right) * \sin \left(q_{1}+q_{2}\right)\right) * d q_{1} \\
& H_{22}=\left(m_{1} * g * l_{2} * \cos \left(q_{1}+q_{2}\right)+0.5 * m_{2} * g * l_{2} * \cos \left(q_{1}+q_{2}\right)+m_{6} * g * l_{2} * \cos \left(q_{1}+q_{2}\right)\right. \\
& +m_{3} * g * l_{2} * \cos \left(q_{1}+q_{2}\right)+m_{4} * g *\left(l_{2}-0.5 * r_{3}-0.5 * l_{5} * \cos \left(q_{1}+q_{2}\right)\right. \\
& +m_{5} * g *\left(l_{2}-0.5 * r_{4} * \cos \left(q_{1}+q_{2}\right)\right)
\end{aligned}
$$

The vector of gravitational torque is,

$$
G(q)=\left[\begin{array}{ll}
G_{1} & G_{2}
\end{array}\right]^{T}
$$

while $i=1$, corresponding to the model of swing phase:

$$
\begin{aligned}
G_{1} & =0.5 * m_{1} * g * l_{3} * \cos \left(q_{1}\right)+m_{2} * g * l_{3} * \cos \left(q_{1}\right)+0.5 * m_{2} * g * l_{4} * \cos \left(q_{1}+q_{2}\right) \\
& +0.5 * m_{3} * g * l_{3} * \cos \left(q_{1}\right)+0.5 * m_{3} * g * r_{4} * \cos \left(q_{1}+q_{2}\right) \\
& +0.5 * m_{4} * g * r_{2} * \cos \left(q_{1}\right)+0.5 * m_{5} * g *\left(l_{3}-r_{1}\right) * \cos \left(q_{1}\right) \\
& +0.5 * m_{5} * g * r_{3} * \cos \left(q_{1}+q_{2}\right)-0.5 * m_{5} * g * l_{5} * \sin \left(q_{1}+q_{2}\right) \\
G_{2} & =0.5 * m_{2} * g * l_{4} * \cos \left(q_{1}+q_{2}\right)+0.5 * m_{3} * g * r_{4} * \cos \left(q_{1}+q_{2}\right) \\
& +0.5 * m_{5} * g * r_{3} * \cos \left(q_{1}+q_{2}\right)+0.5 * m_{5} * g * l_{5} * \sin \left(q_{1}+q_{2}\right)
\end{aligned}
$$

while $i=2$, corresponding to the model of stance phase:

$$
G_{1}=G_{2}=0.1
$$




\section{Appendix B. Kinematics Analysis}

The simulation experiment at the end of this article shows the contraction change of the pneumatic muscles when the joints motion. The calculation process is based on the following content:

By analysis of the exoskeleton structure, the relationships between the length of the pneumatic muscles and the joint angles are:

$$
\begin{aligned}
\text { length } & =\operatorname{sqrt}\left(l_{11}^{2}+l_{12}^{2}-2 * l_{11} * l_{12} * \cos \left(q_{1}\right)\right) \\
\text { length } & =\operatorname{sqrt}\left(l_{21}^{2}+l_{22}^{2}-2 * l_{21} * l_{22} * \cos \left(q_{2}\right)\right) \\
\text { length } & =\operatorname{sqrt}\left(l_{41}^{2}+l_{31}^{2}+l_{32}^{2}+2 * l_{31} * l_{32} * \cos \left(q_{2}\right)\right. \\
& \left.+2 * l_{41} * \operatorname{sqrt}\left(l_{31}^{2}+l_{32}^{2}+2 * l_{31} * l_{41} * \cos \left(q_{2}\right) * \cos \left(q_{1}+\text { alpha }\right)\right)\right)
\end{aligned}
$$

where,

$$
\text { alpha }=\operatorname{arcos} \frac{2 * l_{31}^{2}+l_{32}^{2}+2 * l_{31} * l_{32} * \cos \left(q_{2}\right)}{2 * l_{31} * \operatorname{sqr}\left(l_{31}^{2}+l_{32}^{2}+2 * l_{31} * l_{32} * \cos \left(q_{2}\right)\right)}
$$

$l_{11}=l_{2}, l_{12}=r_{2}, l_{21}=\left(l_{3}-r_{1}\right)+r_{3} / \cos \left(q_{2}\right), l_{22}=l_{5}-r_{3} * \tan \left(q_{2}\right), l_{31}=l_{3}, l_{32}=r_{4}, l_{41}=l_{1}$, their specific values can be found in Table 2 of main text. Approximately, original length of pneumatic muscle as follows:

For muscle 1: when the hip joint angle is 108.764 degree, it is considered that the pneumatic muscle 1 is in a natural state:

$$
\text { length }_{\text {origin } 1}=\operatorname{sqrt}\left(l_{11}^{2}+l_{12}^{2}-2 * l_{11} * l_{12} * \cos (108.764)\right)
$$

For muscle 2: when the knee joint angle is 70 degree, it is considered that the pneumatic muscle 2 is in a natural state:

$$
\text { length } \text { origin } 2=\operatorname{sqrt}\left(l_{21}^{2}+l_{22}^{2}-2 * l_{21} * l_{22} * \cos (70)\right)
$$

For muscle 3: when the hip joint angle is 93.1 degree and the knee joint angle is 0 degree, it is considered that the pneumatic muscle 2 is in a natural state:

$$
\begin{aligned}
& \text { alpha }=\operatorname{arcos} \frac{2 * l_{31}^{2}+l_{32}^{2}+2 * l_{31} * l_{32} * \cos (0)}{2 * l_{31} * \operatorname{sgrt}\left(l_{31}^{2}+l_{32}^{2}+2 * l_{31} * l_{32} * \cos (0)\right)} \\
& \text { length } \text { origin } 3=\operatorname{sqrt}\left(l_{41}^{2}+l_{31}^{2}+l_{32}^{2}+2 * l_{31} * l_{32} * \cos (0)\right. \\
& \left.+2 * l_{41} * \operatorname{sqrt}\left(l_{31}^{2}+l_{32}^{2}+2 * l_{31} * l_{41} * \cos (0) * \cos (93.1+\text { alpha })\right)\right)
\end{aligned}
$$

The relationships between joint angles and pneumatic muscles contraction rates are shown as:

$$
\begin{aligned}
x i_{1} & =1-\operatorname{sqrt}\left(l_{11}^{2}+l_{12}^{2}-2 * l_{11} * l_{12} * \cos \left(q_{1}\right)\right) / \text { length } \\
x i_{2} & =1-\operatorname{sgrtigin} 1 \\
\left.x i_{21}^{2}+l_{22}^{2}-2 * l_{21} * l_{22} * \cos \left(q_{2}\right)\right) / \text { legth } & =1-\operatorname{sqrigin} 2 \\
& +2 * l_{41}^{2}+l_{31}^{2}+l_{32}^{2}+2 * l_{31} * l_{32} * \cos \left(q_{2}\right) \\
& \left.+\operatorname{sqrt}\left(l_{31}^{2}+l_{32}^{2}+2 * l_{31} * l_{41} * \cos \left(q_{2}\right) * \cos \left(q_{1}+\text { alpha }\right)\right)\right) / \text { length }_{\text {origin } 3}
\end{aligned}
$$

\section{References}

1. Kazerooni, H.; Steger, R.; Huang, L. The Berkeley LOWER extremity Exoskeleton; Sage Publication Inc.: Thousand Oaks, CA, USA, 2006; pp. 561-573.

2. Robert, B. Exoskeletons and robotic prosthetics: A review of recent development. Ind. Robot 2009, 36, 421-427.

3. Toyama, S.; Yamamoto, G. Development of wearable- agri- robot mechanism for agricultural work. In Proceedings of the IEEE International Conference on Intelligent Robots and Systems, St. Louis, MO, USA, 10-15 October 2009, pp. 5801-5806.

4. Gilwhoan, C.; Jincheol, H.; Dong, H. The experiments of wearable robot for carrying heavy-weight objects of shipbuilding works. In Proceedings of the IEEE International Conference on Automation Science and Engineering, Taipei, Taiwan, 18-22 August 2014, pp. 978-983. 
5. Kawamoto, H.; Lee, S.; Kanbe, S. Power assist Method for HAL-3 using EMG-based feedback controller. In Proceedings of the IEEE International Conference on Systems, Man and Cybernetics, Washington, DC, USA, 5-8 October 2003, pp.1648-1653.

6. He, Y.; Nathan, K.; Venkatakrishnan, A.; Rovekamp, R. An integrated neuro-robotic interface for stroke rehabilitation using the NASA X1 powered lower limb exoskeleton. In Proceedings of the IEEE International Conference on Medicine and Biology Society, Chicago, IL, USA, 26-30 August 2014, pp. 3985-3988.

7. Toyama, S.; Yamamoto,G. Development of wearable- agri- robot mechanism for agricultural work. J. Abbr. 2008, 10, 142-149.

8. Jezernik, S.; Colombo, G.; Morari, M. Automatic Gait-pattern Adaptation Algorithms for Rehabilitation with a 4-DOF Robot orthosis. IEEE Robot. Autom. 2004, 20, 574-582. [CrossRef]

9. Stegall, P.; Motard, E.; Verschueren, J.P. Rehabilitation exoskeleton design: Exploring the effect of the anterior lunge degree of freedom. IEEE Trans. Robot. 2013, 29, 838-846. [CrossRef]

10. Aaron, J.Y.; Daniel, P.F. State-of-the-art and future directions for robotic lower limb exoskeletons. IEEE Neural Syst. Rehabil. 2016, 25, 1-10.

11. Tucker, M.R.; Olivier, J.; Pagel, A.; Bleuler,H. Control strategies for active lower extremity prosthetics and orthotics: A review. J. Neureng. Rehabil. 2015, 12, 1-29. [CrossRef]

12. Yan, T.; Cempini, M.; Oddo, C.M.; Vitiello, N. Review of assistive strategies in power lower-limb orthoses and exoskeletons. Robot. Auton. Syst. 2015, 64, 120-136. [CrossRef]

13. Sankai, Y. HAL: Hybrid Assistive Limb Based on Cybernics. In Proceedings of the Isrr 2007 Robotics Reserarch-The International Symposium, Hiroshima, Japan, 26-29 November 2007; pp. 25-34.

14. Kazerooni,H.; Steger, R.; Huang, L. Hybrid control of the Berkeley lower extremity exoskeleton (BLEEX). Int. J. Robot. Res. 2006, 25, 561-573. [CrossRef]

15. Guo, Q.; Li, S.J.; Jiang, D. Lower extremity exoskeleton: Human-machine coupled modeling, robust control design, simulation, and overload-carrying experiment. Math. Probl. Eng. 2015, 1, 1-15. [CrossRef]

16. Long, Y.; Chen, Z.J. Hybrid control scheme of a hydraulically actuated lower extremity exoskeleton for Load-Carrying. J. Intell. Robot. Syst. 2018, 91, 493-500. [CrossRef]

17. Li, Z.Y.; Dong, W.; Wang, L.K. Lower Limb Exoskeleton Hybrid Phase Control Based on Fuzzy Gain Sliding Mode Controller. J. Abbr. 2008, 10, 142-149.

18. Hong, C.K.; Chang, H.S.; Young, J.S. Locomotion Control Strategy of Hydraulic Lower Extremity Exoskeleton Robot. J. Abbr. 2008, 10, 142-149.

19. Mefoued, S. A second order sliding mode control and a neural network to drive a knee joint actuated orthosis. Neurocomputing 2014, 155, 71-79. [CrossRef]

20. Liu, J.K.; Sun, F.C. Research and development on theory and Algorithms of sliding mode control. Control Theory Appl. 2007, 24, 407-418.

21. Ren, B.; Luo, X.R.; Chen, J.Y. Single Leg Gait Tracking of Lower Limb Exoskeleton Based on Adaptive Iterative Learning Control. Appl. Sci. 2019, 9, 2251-2261. [CrossRef]

22. Zhang, Y.; Wang, J.Z. A model-free control method for estimating the joint angles of the knee exoskeleton. Adv. Mech. Eng. 2018, 10,1-10. [CrossRef]

23. Jin, X.L.; Zhu, S.Q. Single-input adaptive fuzzy sliding mode control of the lower extremity exoskeleton based on human-robot interaction. Adv. Mech. Eng. 2017, 9, 1-9. [CrossRef]

24. Long, Y.; Du, Z.J.; Cong, L. Active disturbance rejection control based human gait tracking for extremity rehabilitation exoskeleton. ISA Trans. 2017, 67, 389-397. [CrossRef]

25. Zhao, L.; Cheng, H.Y.; Wang, T. Sliding mode control for a two-joint coupling nonlinear system based on extended state observer. J. Abbr. 2018, 73, 20-30. [CrossRef]

26. Cristian, C.; Marco, C.; Giuseppe, C. Design and numercial characterization of a new leg exoskeleton for motion assistance. Robotica 2016, 33, 1147-1162.

27. Mantian, L.; Jing, D.; Fusheng, Z. Towards Online Estimation of Human Joint Muscular Torque with a Lower Limb Exoskeleton Robot. Appl. Sci. 2018, 8, 1610-1627.

28. Abdul, R.A.H.; Aladin, Z.; Begg, R.K.; Yufridin, W. Foot Plantar Pressure Measurement System: A Review. Sensors 2012, 12, 9884-9912. [CrossRef] [PubMed] 
29. Liu, J.M. Establishment of National Standard about Inertial Parameters of Chinese Adults. Ph.D. Thesis, Beijing Sport University, Beijing, China, 2004.

30. Cheng, C.K; Chen, H.H.; Chen, C.S. Segment inertial properties of Chinese adults determined from magnetic resonance imaging. Clin. Biomech. 2000, 15, 559-566. [CrossRef]

(C) 2019 by the authors. Licensee MDPI, Basel, Switzerland. This article is an open access article distributed under the terms and conditions of the Creative Commons Attribution (CC BY) license (http:/ / creativecommons.org/licenses/by/4.0/). 